\title{
THE PREVALENCE OF DENTAL INJURIES IN RUGBY PLAYERS AND THEIR ATTITUDE TO MOUTHGUARDS
}

\author{
R. M. DAVIES, B.D.S., Ph.D., D. BRADLEY, B.D.S., R. W. HALE, B.D.S., \\ W. R. E. LAIRD, F.D.S. and P. D. THOMAS, F.D.S., M.Sc.
}

University of Manchester Dental Hospital, Bridgeford St., Manchester

\section{INTRODUCTION}

Although dental injuries may be considered relatively insignificant when compared to other forms of injury sustained by Rugby players (Weightman and Browne, 1974; Walkden, 1975) they can nevertheless cause considerable discomfort and necessitate the provision of otherwise unnecessary and expensive dental treatment. A certain number of injuries are inevitable in contact sports, but as has been shown in a study of American footballers the risk of dental injury may be minimised by the wearing of mouthguards. Prior to 1962 dental injuries accounted for more than 25-30\% of all injuries in American footballers. Since that time, the mandatory wearing of facemasks and mouthguards has prevented an estimated 100,000 oral injuries annually (Bureau of Dental Health Education Council on Dental Materials and Devices, 1973). The extent to which each of these devices contributed to this reduction in injury is not clear.

In Rugby Football the decision to wear a mouthguard or not is left to the individual, who it is assumed can make an informed decision. The extent and type of dental injuries in Rugby players is poorly documented but available data certainly suggest that the risk of receiving such injuries is high. Hawke and Nicholas (1969) reported that in a sample of 152 New Zealand Rugby players $26 \%$ had suffered injuries to the teeth and $62 \%$ had suffered injuries to either teeth, lips, tongue, jaws or temperomandibular joint. In a similar study of 100 Lancashire Rugby League players, $33 \%$ had received injuries to anterior teeth and lacerations to the cheeks and lips (Clegg, 1969). Despite this evidence the percentage of Rugby players wearing mouthguards is still unsatisfactory (Walkden, 1975). It was decided, therefore, to gain further information as to the prevalence and type of injuries to the teeth received by Rugby players and their attitude to the wearing of mouthguards.

\section{METHODS}

During 1976281 Rugby players from 24 clubs in the North of England agreed to participate in a formal interview conducted by two of the authors (DB and
RH). Each subject was asked a number of precoded and open questions pertaining to dental injuries received while playing Rugby and their attitude to mouthguards. The answers were recorded on a standardised form.

The study was restricted to 1 st $X V$ and 3 rd $X V$ players since it was considered that this would provide a fair representation of the different standards of Rugby football.

\section{RESULTS}

\section{Teeth lost}

$43(15 \%)$ of those interviewed reported that they had lost teeth as a result of playing Rugby and of those 12 (28\%) had lost teeth on more than one occasion.

A total of 82 teeth had been lost. Of the players affected, $24(56 \%)$ had lost one tooth; $19(44 \%)$ two or more. The teeth most frequently affected were the upper incisors (58\%) and upper canines and premolars (17\%). Tooth loss was less common in the lower jaw (18\%) but an interesting finding was that molar teeth, both upper and lower, were lost in $14 \%$ of cases. The mean age of a player at the time teeth were lost was 21.2 years. The team played for when injury resulted in tooth loss was; 1 st XV (49\%), School (28\%), 2nd XV (14\%), 3rd, 4th and 5 th XV $(5 \%)$, County $(2 \%)$, International (2\%).

The position of players on the field when they received injury of the teeth was; Front five $(42 \%)$, Back row $(30 \%)$, Threequarter $(14 \%)$, Halfback $(9 \%)$, Fullback $(5 \%)$.

\section{Teeth fractured}

$108(38 \%)$ of those interviewed reported that they had teeth broken, but not extracted, as a result of Rugby injury, and of those 31 (29\%) had fractured teeth on more than one occasion. A total of 189 teeth had been fractured, $64(59 \%)$ having fractured one tooth; 44 $(41 \%)$ two or more. As before the teeth most frequently affected were the upper incisors (55\%); upper canines and premolars $(10 \%)$, lower incisors $(13 \%)$, upper molars $(10 \%)$, lower molars $(10 \%)$. The position of the players 
on the field at the time of injury was; Front five (37\%), Back row (29\%), Threequarter (18\%), Halfback (13\%), Fullback (4\%).

\section{Mouthguards}

$67(24 \%)$ of those interviewed wore a mouthguard regularly; 36 (55\%) were provided by a dentist, 30 (44\%) had been purchased at a shop and 1 by post. 33 (49\%) of those wearing a mouthguard had done so only after receiving some form of injury to the teeth. None of the players suffered a dental injury while wearing a mouthguard.

64 (22\%) of players stated that they had worn a mouthguard at some time during their playing career but had ceased to do so. The following reasons were given for having stopped wearing a mouthguard: uncomfortable (37\%); interfered with breathing (29\%), loose $(19 \%)$, lost $(10 \%)$, interefered with speech $(1 \%)$, other (4\%). $66 \%$ of players said that they would be willing to wear a mouthguard if provided with one; $34 \%$ said they would be unwilling. Their reasons for not wearing a mouth guard were interference with breathing (37\%), not worried about injury (16\%), might swallow it (11\%), interfere with speech $(9 \%)$, cost $(7 \%)$, other $(20 \%)$. Of those willing to wear one $66 \%$ would pay up to $£ 5,17 \%$ up to $£ 10,17 \%$ nothing.

\section{DISCUSSION}

The prevalence and type of dental injury may be summarised as follows:-

Total number of players with injuries Players with lost and fractured teeth Players with lost but no fractured teeth Players with fractured but no lost teeth Players with no injury
$125(45 \%)$

$24(9 \%)$

$19(6 \%)$

$84(30 \%)$

$154(55 \%)$
These data confirm the findings of Hawke and Nicholas (1969) and Clegg (1969) that a high proportion of Rugby players suffer some form of injury to the teeth during their playing career. The risk of dental injury appears to be unrelated to different playing positions when expressed on an individual basis but is related to the standard at which the game is played. It should be emphasised that the present study underestimates the total extent of damage since lacerations of soft tissue and fractures of the jaw were not included in this survey. Other teeth may also have become non-vital, but not suffered visible damage. Nevertheless, the extent of injury in Rugby players should be the cause of some concern for three reasons. Firstly, the individual may suffer discomfort, aesthetic disfigurement and a reduction in masticatory function. Secondly, the different types of injury require treatment procedures of varying degrees of complexity including the provision of removable or fixed appliances, crowns or simple restorations. Thirdly, dental injuries and their consequences could be almost totally prevented by the wearing of mouthguards.

Traditionally mouthguards are divided into three types; preformed, mouth formed and individually made. This study has clearly shown that in order to be acceptable a mouthguard should be comfortable to wear and easily retained; it should not interfere with breathing or speech. Utilising such criteria there is little doubt that the most acceptable and desirable form of mouthguard would be the individually made type of vinyl plastic (Nachman et al, 1965; Dennis and Parker, 1972). In addition to preventing direct and indirect injuries to the teeth, this type of mouthguard will also protect the lips and cheeks from laceration against the teeth, absorb forces which might fracture the mandibular angle or condyle and instil a greater degree of confidence in players (Stevens, 1972). For these reasons and together with the increasing popularity of Rugby football, particularly at the junior level, the wearing of mouthguards should be encouraged prior to rather than after injury has occurred.

\section{ACKNOWLEDGEMENTS}

We should like to thank the players who participated in this study and Miss E. Laws, who helped in the design of the questionnaire and processed the data.

\section{REFERENCES}

Bureau of Dental Health Education, Council on Dental Materials and Devices, 1973. Mouth protectors: eleven years later. Journal of the American Dental Association, 86, 1365-1367.

Clegg, J. H. 1969, Mouth protection for the Rugby football players. British Dental Journal, 127, 341.

Dennis, C. G. and Parker, D. A. S. 1972, Mouthguards in Australian sport. Australian Dental Journal, 17, 228-235.

Hawke, J. G. and Nicholas, W. K. 1969, Dental injuries in Rugby football. New Zealand Dental Journal, 65, 173-175. 
Nachman, B. M., Smith, J. F. and Richardson, J. F. 1965, Football players' opinions of mouthguards. Journal of the American Dental Association, 70, 62.

Stevens, O. O. 1972, Prevention of traumatic dental and oral injuries of the teeth. C. V. Mosby Company, St. Louis, 299.

Walkden, L. 1975, The medical hazards of Rugby Football. The Practitioner, 215, 201-207.

Weightman, D. and Browne, D. M. 1974, Injuries in Association and Rugby Football. British Journal of Sports Medicine, 8, 183-187. 